\title{
Kaderschmieden der Wirtschaft und/oder Universitäten? Der Auftrag der Wirtschaftsuniversitäten und -fakultäten im 21. Jahrhundert
}

\author{
Gebhard Kirchgässner*
}

Universität St. Gallen

\section{Einleitung}

Es gibt im deutschsprachigen Raum heute drei Wirtschaftsuniversitäten, Mannheim, St. Gallen und Wien, wobei sich Mannheim zumindest in ihrem Fächerspektrum inzwischen am weitesten einer klassischen Universität angenähert hat. Worin aber unterscheiden sich diese Wirtschaftsuniversitäten und - in geringerem Maße - auch die Wirtschaftsfakultäten von anderen Universitäten bzw. Fakultäten, so dass es Sinn macht, auf die Herausforderungen, die sich für sie stellen, gesondert einzugehen? Weshalb gibt es überhaupt bzw. weshalb entstanden Wirtschaftsuniversitäten? Sieht man einmal vom Spezialfall der theologischen Hochschulen ab, die üblicherweise (oder sogar ausschließlich) nicht in öffentlicher Trägerschaft sind, gibt es neben den Wirtschaftsuniversitäten im öffentlichen Hochschulwesen der deutschsprachigen Länder nur zwei Bereiche, für die eigene Hochschulen bzw. Universitäten gegründet wurden: die Technik und die Medizin. In allen drei Fällen stand bei der Gründung, die bei den technischen Hochschulen teilweise bereits in der ersten Hälfte des neunzehnten Jahrhunderts, ${ }^{1}$ bei den Wirtschaftshochschulen Ende des 19. bzw. Anfang des 20. Jahrhunderts erfolgte, die Ausbildung im Vordergrund, seien es Techniker, Ärzte oder Kaufleute. ${ }^{2}$ Im Gegensatz zu den traditionellen Universitäten, die (zumindest ihrem Anspruch nach) in dieser Zeit dem Humboldtschen Ideal der Einheit von Forschung und Lehre verpflichtet waren, stand bei den Wirtschafts- bzw. Handelshochschulen zu Beginn die Lehre im Vordergrund: Sie wurden zumeist von Handelsstädten gegründet, in denen die Kaufmannschaft ihrem Nachwuchs eine entsprechende Ausbildung zukommen lassen wollte.

Diese Konzentration auf die Lehre, insbesondere auf die Ausbildung von Kaufleuten, hat, wie unten noch zu zeigen sein wird, bis heute Konsequenzen, auch

\footnotetext{
*Korrespondenzadresse: Universität St. Gallen, SIAW-HSG, Bodanstrasse 8, CH-9000 St. Gallen, Schweiz, E-Mail: Gebhard.Kirchgaessner@unisg.ch. - Überarbeitete schriftliche Fassung eines Vortrags im Rahmen des Workshops „Der Auftrag der Universitäten im 21. Jahrhundert“ der Österreichischen Forschungsgemeinschaft in Baden bei Wien, 11. Dezember 2010. Für Unterstützung bei der historischen Recherche danke ich Frau Teresa Körner, M.A. - Die hier vertretenen Auffassungen sind ausschließlich jene des Autors; sie decken sich nicht notwendigerweise mit den offiziellen Positionen der Universität St. Gallen. Eine frühere Fassung findet sich unter http://www.oefg.at/text/veranstaltungen/ universitaeten/Beitrag_Kirchgaessner.pdf.

1. Damals sogenannte 'Polytechnische Schulen' wurden in Berlin 1821, Karlsruhe 1825, München 1827 und Hannover 1831 gegründet. Die ETH Zürich wurde 1855, die RWTH Aachen 1870 gegründet.

2. Der Vorrang der Lehre gilt (in vielleicht noch stärkerem Maße) für die privat (z.B. von Orden) betriebenen theologischen Hochschulen.
} 


\section{Gebhard Kirchgässner}

wenn die Forschung heute auch in den Wirtschaftsuniversitäten ein wesentlicher Bereich ist. Sie zeigt sich zum einen in der Struktur der Universität, die eindeutig von der Betriebswirtschaftslehre dominiert wird: An der Universität St. Gallen unterrichteten z.B. zu Beginn des Jahres 201048 Prozent der Professoren, 64 Prozent der Dozenten sowie 57 Prozent der Lehrbeauftragten dieses Fach, und unter den Studierenden der Bachelorstufe studierten im Herbstsemester 200962.2 Prozent Betriebswirtschaftslehre. Die Volkswirte machten dagegen nur 12 Prozent der Bachelorstudierenden aus. ${ }^{3}$ Ganz ähnlich sieht es an der Wirtschaftsuniversität Wien aus. Dort studieren 51 Prozent Betriebswirtschaft, 36 Prozent Handelswissenschaft, 7 Prozent Wirtschaftspädagogik und gerade noch 6 Prozent Volkswirtschaft. ${ }^{4}$ Wegen des erweiterten Fächerspektrums sieht es in Mannheim insgesamt etwas anders aus; unter allen Studierenden gehörten ,nur 38.2 Prozent zur betriebswirtschaftlichen Fakultät. Betrachtet man dagegen nur die Wirtschaftsstudenten, dann macht der Anteil der Betriebswirte in Mannheim 81.7 Prozent aus. ${ }^{5}$ Dabei nahm der Anteil der Wirtschaftsstudenten an der gesamten Studentenschaft in der jüngsten Vergangenheit zu und wird vermutlich weiter zunehmen. In der Schweiz ist die Zahl der Wirtschaftsstudenten seit 1999 um 3.9 Prozent pro Jahr gestiegen, während die Zahl aller Studierenden nur um 2.6 Prozent gestiegen ist. Dementsprechend ist auch ihr Anteil an der gesamten Studentenschaft von 13.2 auf 14.2 gestiegen. ${ }^{6}$ In Deutschland, wo das Wirtschaftsstudium nicht ganz so populär ist, ist der Anteil der Betriebswirte an der gesamten Studentenschaft seit dem Jahr 1991 (nach der Vereinigung) von 7.0 auf 8.2 Prozent angestiegen. $^{7}$ Nur in Österreich hat der Anteil der Wirtschaftsstudierenden nach einem Anstieg zu Beginn der neunziger Jahre in den letzten Jahren abgenommen. Er betrug im Studienjahr 1990/91 20.7 Prozent, im Studienjahr 2000/01 23.6 Prozent, aber im Studienjahr 2009/10 nur noch 19.1 Prozent der Studierenden. ${ }^{8}$ Dennoch haben wir es auch in Österreich in den Wirtschaftswissenschaften im Bereich der Lehre mit einem Massenphänomen zu tun: Den 23'500 Studierenden stehen an der Wirtschaftsuniversität Wien heute gerade einmal 80 Professorinnen und Professoren gegenüber. ${ }^{9}$

An einer Universität sollte neben der Lehre auch die Forschung nicht zu kurz kommen. Beim Massenbetrieb in der Lehre ist dies nicht einfach. Dabei ist freilich zwischen der Volks- und der Betriebswirtschaftslehre zu unterscheiden. In der traditionell eher an den (traditionellen) Universitäten beheimateten Volkswirtschaftslehre gibt es eine Forschungstradition, die sich von derjenigen in

3. Siehe http://www.presse.unisg.ch/ /media/Internet/Content/Dateien/UeberUns/HSGMediacorner/ Aktuell/ 2009/Dezember2009/HSG_Studierendenstatistik_23Dezember2009.ashx?fl=de (07/12/10).

4. Siehe http://www.portal.ac.at/owa/portal.details?ogn_id_in=19 (07/12/10).

5. Dies gilt für den Stichtag des 30. April 2009. Siehe hierzu http://www.uni-mannheim. de/1/universitaet/ profil/zahlen_geschichte/statistiken/fss_2009/statistik_fss09.pdf (07/12/10).

6. Siehe Statistisches Jahrbuch der Schweiz 2010, elektronische Version, Tabelle T 15.2.1.4.5.

7. Siehe http://www.destatis.de/jetspeed/portal/cms/Sites/destatis/Internet/DE/Content/Statistiken/ Bildung ForschungKultur/Hochschulen/Tabellen/Tabellenuebersicht,templateId=renderPrint.psml\# LangeReihen Studierende (07/12/10).

8. Diese Zahlen gelten freilich für die gesamten Wirtschafts- und Sozialwissenschaften; die Zahlen für die Wirtschaftswissenschaften bzw. die Betriebswirtschaftslehre werden nicht gesondert ausgewiesen. Siehe http://www.statistik.at/web_de/statistiken/bildung_und_kultur/formales_bildungswesen/ universitaeten_studium/index.html (07/12/10).

9. Siehe www.portal.ac.at/owa/portal.details?ogn_id_in=19 bzw. http://www.wu.ac.at/structure/ departments/ faculty/professors (07/12/10). 


\section{Kaderschmieden der Wirtschaft und/oder Universitäten?}

den anderen Wissenschaften kaum unterscheidet. Dort sind freilich auch die Zahlen der Studierenden überschaubar; zumindest im Haupt- bzw. im Masterstudium. ${ }^{10}$ In der zunächst vorwiegend auf die Lehre ausgerichteten, vor allem in den Wirtschaftshochschulen beheimateten Betriebswirtschaftslehre war diese Tradition sehr viel schwächer. Eine internationale Anerkennung als Business School ist heute freilich ohne international anerkannte Forschung nicht mehr möglich. So bewertet die Financial Times in ihrem Ranking der Master of Business Administration (MBA) und der Executive MBA (EMBA) neben der Ausbildung auch die Forschung an den jeweiligen Institutionen. Neben der Belastung in der Lehre besteht daher eine zweite Herausforderung darin, die Forschung zu stärken.

Damit sind die Aufgaben einer Wirtschaftsuniversität noch nicht vollständig umschrieben. Zu Forschung und Lehre kommen zwei weitere Bereiche: Die Beratung und die Weiterbildung. In beiden Feldern sind die Wirtschaftswissenschaftler weit stärker als die Vertreter der traditionellen Geistes-, Sozial- und Naturwissenschaften tätig. Dies wird auch von ihnen erwartet, sei es (bei den Volkswirten) von der Politik, sei es (bei den Betriebswirten) von der Wirtschaft. In beiden Feldern haben sie freilich kein Monopol, sondern müssen zum Teil hart um ihre Position kämpfen. Damit ergeben sich insgesamt (mindestens) vier zentrale Bereiche, in denen sie Herausforderungen gegenüberstehen: (i) Die akademische Lehre, (ii) die wissenschaftliche Forschung, (iii) die Beratung sowie (iv) die Weiterbildung. Dabei ist zu berücksichtigen, dass sich diese Herausforderungen für die Volkswirtschaftslehre teilweise anders stellen als für die Betriebswirtschaftslehre.

Im Folgenden soll auf diese vier Bereiche eingegangen werden. Dabei werden wir uns auf den deutschsprachigen Raum beschränken; im angelsächsischen Raum stellen sich manche Probleme deutlich anders. Dies bedeutet freilich nicht, dass dieser Raum abgeschottet wäre und für sich allein betrachtet werden könnte: Ein erheblicher Teil der Herausforderungen, denen sich heute die Wirtschaftswissenschaften auch im deutschsprachigen Raum gegenüber sehen, ergibt sich aus der Globalisierung der Wissenschaften und dem dadurch entstandenen und sich weiter verschärfenden internationalen Wettbewerb, der gerade auch in den Wirtschaftswissenschaften besonders intensiv ist. Er wirkt sich auf alle vier Bereiche unserer Tätigkeit aus: Wir stehen im internationalen Wettbewerb nicht nur im Forschungsbetrieb, sondern auch um gute Studierende, um zahlungskräftige Teilnehmer in den Weiterbildungskursen sowie auch bei der wirtschaftpolitischen Beratung. Dabei sind unter den Konkurrenten nicht nur die Consulting-Firmen international tätig, sondern auch durch öffentliche Gelder (weitgehend) finanzierte Wirtschaftsforschungsinstitute. So waren z.B. bei der Gemeinschaftsdiagnose für die Entwicklung der deutschen Wirtschaft im Oktober 2010 neben deutschen Wirtschaftsforschungsinstituten auch die Konjunkturforschungsstelle der ETH Zürich sowie das Institut für Höhere Studien in Wien engagiert. ${ }^{11}$

10. Eine erhebliche Belastung ergibt sich in der Lehre freilich dadurch, dass Volks- und Betriebswirte zu Beginn ihres Studiums gemeinsame Veranstaltungen besuchen; traditionell war das Studium bis zum Vordiplom häufig sogar identisch, so dass die Volkswirte auch Massenveranstaltungen zu betreuen hatten (und haben).

11. Siehe http://www.ifw-kiel.de/wirtschaftspolitik/konjunkturprognosen/konjunkt/2010/gdherbst2010.pdf (24/01/11). - Zur Situation der Universitäten im sich verstärkenden internationalen Wettbewerb siehe auch Frey (1997). 


\section{Gebhard Kirchgässner}

Davor soll kurz auf die Geschichte der Wirtschaftsuniversitäten eingegangen werden (Abschnitt 2). Dies ist sinnvoll, um die heutige Situation zu verstehen. Danach werden die akademische Lehre (Abschnitt 3) sowie die wissenschaftliche Forschung betrachtet (Abschnitt 4). In diesen Bereichen stellen sich die Anforderungen sehr unterschiedlich. Berücksichtigt man z.B., dass nicht nur das Schwergewicht der Lehre bei der Betriebswirtschaftslehre liegt, sondern dass auch die Volkswirte ihre Lehre wesentlich für Studenten der Betriebswirtschaftslehre erbringen, stellt sich die Frage, wie die Volkswirtschaftslehre an einer Wirtschaftsuniversität ausgerichtet sein soll. Bei der in Abschnitt 5 behandelten Beratung ist zwischen der wirtschaftspolitischen Beratung durch die Volkswirte und der Beratung von Unternehmen durch die Betriebswirte zu unterscheiden. Die in Abschnitt 6 diskutierte Weiterbildung schließlich ist im Wesentlichen eine Domäne der Betriebswirte (und auch der Juristen); die Volkswirte spielen hier nur am Rande mit. Die Arbeit schließt mit dem Verweis auf ethische Probleme, mit denen sich die wirtschaftswissenschaftlichen Fakultäten heute konfrontiert sehen (Abschnitt 7).

Die Probleme, denen sich die Wirtschaftswissenschaften heute stellen müssen und die hier diskutiert werden sollen, sind freilich ähnlich (und möglicherweise teilweise sogar identisch) wie jene, mit denen sich andere Wissenschaften konfrontiert sehen. Dies gilt z.B. für die Herausforderungen, die sich durch die Internationalisierung der Wissenschaften sowie durch die Bologna-Reform ergeben. Wenn im Folgenden die Situation der Wirtschaftswissenschaften beschrieben und analysiert wird, sind Parallelen zu anderen Wissenschaften kaum zu vermeiden.

\section{Zur Geschichte der Wirtschaftsuniversitäten}

Vorläufer von Wirtschaftsfakultäten sowie von Wirtschaftshochschulen gab es im deutschsprachigen Raum bereits im 18. Jahrhundert. Der sächsische Hofund Kommerzienrat Paul Jakob Marperger (1656 - 1730) regte bereits im Jahr 1715 die Errichtung einer kaufmännischen Akademie mit den entsprechenden Lehrfächern an. ${ }^{12}$ Bevor es dazu kam, wurde in Frankreich bereits gehandelt. Schon seit Gründung der Handels- und Industrieakademie in Paris im Jahr 1803 stellte sich dort die Frage nach einer betriebswirtschaftlichen Ausbildung. Als Vorbild diente die bereits 1781 gegründete Handelsschule in Mühlhausen (Elsass). Die erste Handelsakademie wurde schließlich 1819 als ,Ecole Spéciale de Commerce et d'Industrie de Paris' gegründet. ${ }^{13}$

Es dauerte etwa 80 Jahre, bevor es zur Gründung entsprechender Einrichtungen im deutschsprachigen Raum kam; dies geschah erst am Ende des 19. bzw. zu Beginn des 20. Jahrhunderts. Dabei standen weniger universitäre bzw. wissenschaftliche Überlegungen als vielmehr praktische Probleme im Vordergrund: Als im Jahr 1898 die Handelshochschule Leipzig, die Handelsakademie St. Gallen und die k.k. Exportakademie Wien sowie im Jahr 1907 die Handelshochschule Mannheim gegründet wurden, ging es weniger um universitäre Ausbildungen, als vielmehr um eine bessere Ausbildung der jungen Kaufleute. ${ }^{14}$ In einer Zeit des Wirtschaftswachstums sowie zunehmender internationaler Handelsverflechtungen

12. Siehe hierzu Zander (2004, S. 44).

13. Siehe hierzu Fournas (2010, S. 20ff.).

14. Zur Entwicklung in Deutschland siehe Zander (2004), zur Geschichte der Universität St. Gallen Thürer (1974), Hayashima (1995) und Burmeister (1998), zur Geschichte der Wirtschaftsuniversität 
wurde dies immer wichtiger. Ähnliche Überlegungen standen Pate, als z.B. im Jahr 1910 die Stockholm School of Economics ihre Tore für die ersten 110 Studierenden öffnete. ${ }^{15}$ Der Schwerpunkt lag bei diesen Gründungen eindeutig auf der Betriebswirtschaftslehre; Volkswirtschaftslehre wurde schon zuvor und nach diesen Gründungen zunächst auch weiterhin vorwiegend an den (traditionellen) Universitäten unterrichtet.

Es ist daher nicht überraschend, dass sich diese Entwicklung in eigenständigen Institutionen und nicht in den etablierten Universitäten vollzog; man wollte zumindest teilweise auch gar keine Universität sein. ${ }^{16}$ Zwar wurden in dieser Zeit auch an den Universitäten erste Lehrstühle für Betriebswirtschaftslehre eingerichtet, aber sie spielten keine wesentliche Rolle; an den Universitäten dominierte bis nach dem 2. Weltkrieg im Bereich der Wirtschaftswissenschaften eindeutig die Volkswirtschaftslehre. Dies wirkt zumindest an den alten Universitäten bis heute nach. So bilden z.B. in Baden-Württemberg die beiden Universitäten Freiburg und Heidelberg nach wie vor nur Volkswirte aus, und die dritte alte Universität, Tübingen, hat die Betriebswirtschaftslehre als eigenständiges Studienfach erst in den achtziger Jahren des vergangenen Jahrhunderts eingerichtet.

Es dauerte deshalb auch einige Zeit, bevor die neu gegründeten Handelsakademien bzw.-hochschulen akademische Rechte erhielten. Mannheim erhielt das Habilitationsrecht vergleichsweise früh im Jahr 1916 sowie das Promotionsrecht im Jahr 1929, die Wirtschaftsuniversität Wien das Promotionsrecht und damit einen zumindest quasi-universitären Status im Jahr 1930, und St. Gallen erst im Jahr 1938. Die universitäre Forschung spielte zu Beginn und bis in die jüngere Vergangenheit eine untergeordnete Rolle. Mit Ausnahme der Volkswirtschaftslehre in Mannheim galt dies auch noch, als in den Jahren 1967 Mannheim, 1975 Wien und 1996 St. Gallen in Universitäten umgetauft wurden.

Darin unterscheiden sie sich z.B. von den amerikanischen Business Schools. Dort nimmt die Volkswirtschaftslehre seit langem einen wichtigen Platz ein, und sie ist auch entsprechend integriert. An berühmten Institutionen wie z.B. der Sloan School of Management am MIT waren und sind bekannte Professoren der Volkswirtschaftslehre tätig wie z.B. Richard Schmalensee, Thomas Stocker oder Robert Merton. Ähnliches gilt für die Simon Graduate School of Management der University of Rochester oder für die Carnegie Mellon Tepper School of Business, wo unter anderem Finn E. Kydland, Nobelpreisträger des Jahres 2004, sowie Alan H. Meltzer, einer der Begründer der modernen Geldangebotstheorie, unterrichten. Diesem amerikanischen Vorbild hat sich von den Wirtschaftshochschulen, und sie

Wien http://www.wu.ac.at/strategy/history/ (07/12/10). - Kurz nach der Jahrhundertwende wurden auch Handelshochschulen in Köln (1901), Frankfurt (1901), Berlin (1906), München (1910), Königsberg (1915) und Nürnberg (1919) gegründet. Mit Ausnahme der Handelshochschule Königsberg, die mit Ende des 2. Weltkriegs geschlossen wurde, sind diese jedoch alle als Fakultäten in die entsprechenden Universitäten am Ort eingegliedert worden. Die Handelshochschule Mannheim wurde im Jahr 1933 in die Universität Heidelberg eingegliedert, aber im Jahr 1946 wieder als eigenständige Wirtschaftshochschule eröffnet. Siehe hierzu http://www.hhl.de/fileadmin/Fotos/ unis_im_deut_raum.pdf (07/12/10), speziell zu Mannheim Kirchgässner (1988).

15. Siehe hierzu http://www.sseru.org/ucts/index.php?mid=3;9\&pid=153\&lang=en (05/12/10).

16. Zwar entstand im Gegensatz dazu die Sloan School of Management am MIT als Teil der damaligen technischen Hochschule, aber auch dort standen Anforderungen der Ausbildung von Managern bei der Gründung Pate. Siehe hierzu http://mitsloan.mit.edu/ about/history.php (07/12/10). 


\section{Gebhard Kirchgässner}

sind am ehesten mit diesen Institutionen vergleichbar, nur Mannheim frühzeitig angeschlossen. ${ }^{17}$ Heute versuchen freilich alle Wirtschaftsuniversitäten neben der Lehre auch in der internationalen Forschung präsent zu sein, wie aus ihren Leitbildern bzw. Visionen deutlich wird. Die Universität Mannheim will herausragende Forschung in ihren einzelnen Fächern und im interdisziplinären Verbund fördern; Grundlage ist für sie die Einheit von Forschung und Lehre. ${ }^{18}$ Die Universität St. Gallen sieht sich als eine der führenden Wirtschaftsuniversitäten Europas, anerkannt für ihre Lehre auf allen Stufen lebenslangen Lernens und für ihre Forschung in ausgewählten Gebieten großer gesellschaftlicher Relevanz. ${ }^{19}$ Die Wirtschaftsuniversität Wien schließlich ist noch sehr viel konkreter: Sie möchte die führende wirtschafts- und sozialwissenschaftliche Bildungseinrichtung in Österreich sein; im deutschsprachigen Raum möchte sie zu den besten 5, in Europa zu den besten 15 gehören; sie strebt eine hohe Internationalität in Lehre und Forschung an. ${ }^{20}$

\section{Entwicklungen in der Lehre}

Während die Lehre in den Wirtschaftshochschulen zunächst sehr praktisch auf die Ausbildung von Kaufleuten und deren Bedürfnisse ausgerichtet war, hat sie sich im Verlauf der Akademisierung immer mehr jenen Standards angenähert, die für andere Fächer gelten. Volks- und Betriebswirtschaftslehre wurden normale Diplomstudiengänge bzw. Haupt- und/ oder Nebenfächer für Magister- bzw. Staatsexamensstudiengänge, wobei letztere rein zahlenmäßig kaum von Bedeutung waren. In Zeiten, in denen in vielen Fächern der Numerus Clausus eingeführt wurde, war man im Bereich der Betriebswirtschaftslehre eher zurückhaltend; schließlich hatten die Absolventen dieses Fachs - im Gegensatz zu jenen der Staatsexamensstudiengänge - keinerlei auch nur irgendwie gearteten Anspruch darauf, beim Staat eine Beschäftigung zu erhalten. Überdies war es ein vergleichsweise kostengünstiges Studium. Da die Zahl der Lehrenden nicht in gleichem Maße anstieg wie jene der Studierenden, hat dies dazu geführt, dass die Betreuungsrelationen sich seit den siebziger Jahren deutlich verschlechtert haben. Auch wenn sie damit nicht allein standen, haben die Wirtschaftswissenschaften, insbesondere die Betriebswirtschaftslehre, die Probleme der Massenuniversität in besonderer Weise erfahren müssen. Daran hat sich bis heute (noch) nichts geändert.

Eine wesentliche Änderung kam jedoch mit der Bologna-Reform. Hier wurde ein erster Schritt in eine neue Entwicklung getan, indem die drei Stufen Bachelor, Master und Ph.D. eingeführt und klar unterschieden wurden. ${ }^{21}$ Über die formale Einführung dieser Stufen und eine partielle Umgestaltung der Studienpläne hinaus ist hier freilich bis heute wenig geschehen. Bisher scheint es die Regel zu sein, dass

17. Dies war im Wesentlichen ein Verdienst von Heinz König, der über lange Zeit die dominierende Figur in der Mannheimer Volkswirtschaftslehre war. Siehe hierzu z.B. http://www.idw-online.de/de/ news56244 (06/12/10).

18. Siehe http://www.uni-mannheim.de/1/universitaet/profil/leitbild/index.html (07/12/10).

19. Siehe http://www.unisg.ch/de/UeberUns/PortraetUndStruktur/VisionUndLeitbild.aspx (07/12/10).

20. Siehe http://www.wu.ac.at/strategy/targets/ (07/12/10).

21. St. Gallen war vermutlich die erste Universität im deutschsprachigen Raum, die dieses Konzept konsequent umsetzte. Sie hat damit auch bisher die meiste Erfahrung. Ab dem Jahr 2001 konnte man kein traditionelles Studium mehr beginnen, und im Herbst 2006 wurden zum letzten Mal Diplomprüfungen abgenommen. 
fast jeder, der einen Bachelor-Abschluss hat, auch einen Master-Abschluss anstrebt. In der Schweiz wird sogar jedem, der einen Bachelor-Abschluss hat, der Zugang zu einem Masterstudium im gleichen Fach garantiert. Dies hat dazu geführt, dass Deutsche, die in Deutschland wegen zu schlechter Noten nicht zum Masterstudium zugelassen wurden, in großer Zahl in die Schweiz drängten. Dieser Zustand war offensichtlich nicht nachhaltig. Daher wurde jetzt eine Art Numerus Clausus für deutsche Studierende in der Schweiz beschlossen. ${ }^{22}$

Der einzige wirkliche Vorteil der derzeitigen Situation gegenüber früher ist, dass diejenigen, die früher zwar das Vordiplom bestanden haben, aber - aus welchen Gründen auch immer - keinen Diplomabschluss gemacht haben, jetzt einen zumindest nach offizieller Lesart - berufsqualifizierenden Abschluss haben. Eine wirkliche Änderung dürfte aber erst dann eintreten, wenn der Bachelor außerhalb der Universität allgemein als vollwertiger akademischer Abschluss akzeptiert sein wird. Dies ist heute offensichtlich noch nicht der Fall.

In diesem Zusammenhang sollte auch auf die Motivation der Studierenden Rücksicht genommen werden. Wie oben bereits ausgeführt wurde, strebt die weit überwiegende Anzahl der Wirtschaftsstudierenden einen Abschluss in Betriebswirtschaftslehre an. Darunter kann man - zugegeben etwas vereinfachend zwei größere Gruppen ausmachen: (i) jene, denen letztlich nichts besseres einfällt als BWL, weil sie (nicht ganz zu Unrecht) hoffen, damit später auf jeden Fall einen zumindest nicht ganz schlecht bezahlten ,Job' erhalten zu können, und jene, die eine gut bezahlte Karriere im oberen Management einer Firma anstreben. Ein genuines wissenschaftliches Interesse hat nur eine vergleichsweise geringe Minderheit. Dies bedeutet nicht, dass diese Studierenden im Allgemeinen intellektuell weniger qualifiziert als andere wären, aber die Wirtschaftsuniversitäten ziehen mit ihren Studiengängen und insbesondere mit der Betriebswirtschaftslehre nicht unbedingt jene an, die ein sehr starkes Forschungsinteresse haben. ${ }^{23}$ Andererseits sind sie häufig sehr zielstrebig, was insbesondere in der neuen Welt der Bologna-Reform dazu führt, dass viele sehr genau kalkulieren, wo sich mit welchem Aufwand welche Ergebnisse (Noten) erzielen lassen. Wäre der BachelorAbschluss wie in den angelsächsischen Ländern als vollwertiger Abschluss sowohl innerhalb der Universität als auch auf dem Arbeitsmarkt anerkannt, würden sich vermutlich viele unserer Studierenden mit diesem Abschluss zufrieden geben und die Universität verlassen.

Auch wenn wir uns gerne über ihr mangelndes wissenschaftliches Interesse beklagen, ist es dennoch und zwar gerade auch unter diesem Gesichtspunkt unsere Aufgabe, diesen Studierenden eine gute Ausbildung zukommen zu lassen. In einer Zeit, in der die Zahl der Studierenden stark steigt, aber diejenige der Professoren allenfalls sehr schwach zunimmt, wird dies zusehends schwieriger. ${ }^{24}$ Andererseits bietet gerade die Bologna-Reform hier neue Möglichkeiten. Auch wenn es der

22. Siehe hierzu Kaiser, Höhere Hürden für deutsche Studenten, swissinfo.ch vom 24. August 2011, http://www.swissinfo.ch/ger/Home/Archiv/Hoehere_Huerden_fuer_deutsche_Studenten.html?cid= $30980602(31 / 08 / 11)$.

23. Dies schließt nicht aus, dass es möglich ist, bei einigen von ihnen im Verlauf des Studiums ein solches Interesse zu wecken.

24. An der Universität St. Gallen hat sich in den letzten 15 Jahren, d.h. vom Wintersemester 1994/95 bis zum Herbstsemester 2010 die Zahl der Studierenden von knapp 4000 auf 6726, d.h. um etwa 70 Prozent erhöht. Während der Mittelbau stark ausgeweitet wurde, ist gleichzeitig die Zahl der Professorinnen und Professoren mit 71 (gemessen in Vollzeitäquivalenten) konstant geblieben. Siehe 


\section{Gebhard Kirchgässner}

heute (noch) vorherrschenden Meinung widerspricht, ist doch für viele spätere berufliche Tätigkeiten der Bachelor-Abschluss vollständig ausreichend. Dazu müsste er aber auch sinnvoll ausgestaltet werden. Wie dies möglich ist, zeigen uns die Angelsachsen. Die einführenden Texte kommen auch in Volkswirtschaftslehre mit einem Minimum an Mathematik aus; es geht im Wesentlichen darum, wirtschaftliche Zusammenhänge zu erfassen bzw., um es auf die methodische Ebene $\mathrm{zu}$ heben, das Denken in Anreizen zu lernen. ${ }^{25}$ Dies ist alles andere als trivial. Im deutschsprachigen Raum quälen wir die Studierenden dagegen in den ersten Semestern mit einer Mathematik, die sicher 90 Prozent der BWL-Studierenden außerhalb der Universität niemals mehr in ihrem Leben anwenden werden. ${ }^{26}$ Diese Mathematik (sowie Kurse in mikroökonomischer Theorie) verwenden wir als Selektionskriterium, um die Zahl der Studierenden zu reduzieren. Dabei ist nichts dagegen einzuwenden, diejenigen, die für ein Wirtschaftsstudium nicht geeignet sind, relativ frühzeitig darüber $\mathrm{zu}$ informieren. In St. Gallen geschieht dies z.B. dadurch, dass das erste Jahr als Assessmentstufe konzipiert ist, die man bestehen muss, bevor man zu dem anschließenden, 4-semestrigen eigentlichen Bachelorstudium zugelassen wird. Dies dürfte eine sinnvolle Konstruktion sein, aber ob die Selektion wirklich anhand der Mathematikkenntnisse bzw. -fähigkeiten erfolgen soll, darf bestritten werden. ${ }^{27}$ Es gibt genügend Mathematiker (und auch mathematische Ökonomen), denen trotz ihrer ausgefeilten mathematischen Kenntnisse das Denken in Anreizen fremd geblieben ist. ${ }^{28}$

Dies steht nicht im Widerspruch dazu, dass die Mathematik in der wirtschaftswissenschaftlichen Forschung und insbesondere in der Nationalökonomie unverzichtbar ist. Die entsprechende Ausbildung kann aber ohne weiteres und sollte auch auf der Masterstufe erfolgen. ${ }^{29}$ Die Selektion auf dieser Stufe sollte zumindest auch über die analytischen Fähigkeiten erfolgen. Auch wenn dieser Weg sowohl in der Schweiz als auch in Deutschland inzwischen verbaut erscheint: Man kann sich sehr gut ein System vorstellen, in welchem sowohl Universitäten als auch Fachhochschulen zum Bachelor ausbilden, bei der Zulassung zum Masterstudium an einer Universität aber eine Aufnahmeprüfung abgelegt werden muss, wobei einzig deren Resultat zählt, unabhängig davon, wo die erste Ausbildung erfolgt ist. Gesetzt den Fall, es wird ein entsprechendes Stipendiensystem eingeführt, könnte man auf der Masterstufe auch erwägen, zur

hierzu Egger und Dyllick (2010, S. 59), Universität St. Gallen (2011, S. 16, S. 37) sowie Bundesamt für Statistik, Hochschulpersonal 1995, Bern 1996, S. 13. (Nach den derzeitigen Plänen sollen in den nächsten Jahren jedoch 20 neue Professuren geschaffen werden.)

25. Siehe z.B. die klassischen Lehrbücher von Samuelson (1948) oder Mankiw (2009).

26. Kaum jemand, der in die Praxis geht, wird sich z.B. jemals wieder mit dem Problem einer partiellen Integration konfrontiert sehen. Dies gilt im Übrigen auch für viele, die im Bereich der Betriebswirtschaftslehre in der Wissenschaft bleiben.

27. Etwas anders sieht es mit Grundkenntnissen in der Statistik aus, soweit diese noch nicht in der Schule vermittelt wurden. Aber auch hier gibt es Lehrbücher, bei denen der mathematische Aufwand gering ist. Siehe z.B. Yeomans (1968).

28. Die Frage, welche Rolle die Mathematik in der Ausbildung der Ökonomen spielen soll, wird auch in den Vereinigten Staaten seit längerem diskutiert. Siehe hierzu z.B. Butler, Finegan und Siegfried (1994, 1998). Die Verwendung graphischer Darstellungen im Rahmen dieser Lehre wird z.B. von E. Cohn und S. Cohn (1994) diskutiert.

29. Zur Ausbildung von Volkswirten auf der Unterstufe siehe z.B. Frank (2002) oder Hansen, Salemi und Siegfried (2002), zum Graduiertenstudium Colander und Klamer (1987) sowie Kasper (1991) und Krueger (1991). 
Finanzierung Studiengebühren zu erheben, welche den Kosten des Studiums in etwa entsprechen.

Damit ändert sich freilich nichts am Massenproblem auf der Bachelorstufe. Hier ergibt sich eine ganz neue Herausforderung durch die elektronischen Medien. Zunehmend kommen hervorragend konzipierte elektronische, häufig interaktive Materialien für Einführungskurse auf den Markt, welche die traditionelle Großvorlesung durch ein Selbststudium ablösen könnten. ${ }^{30}$ Aufgabe der Universität wäre dann, auf dieser Stufe (nur noch) die Betreuung der Studierenden in kleinen Gruppen in Übungen und Tutorien. In den Grundvorlesungen z.B. für Mikround Makroökonomik wird heute ein weitgehend normierter Stoff vermittelt, wobei häufig auch Lehrbücher aus dem angelsächsischen Raum (bisher oft noch in deutscher Übersetzung) verwendet werden. Zudem dürften auch hier die Vorlesungen zunehmend in englischer Sprache gehalten werden. Unter diesen Bedingungen stellt sich die Frage, inwieweit ein auf begleitetem Selbststudium basierendes System nicht sinnvoller als die traditionelle Großvorlesung ist, bei welcher mehr als 1000 Studierende in einem Raum oder, falls ein so großer Raum nicht verfügbar ist, in mehreren Räumen mit Videoübertragung den Ausführungen eines Professors folgen. Ein persönlicher Kontakt ist in beiden Varianten nicht mehr möglich, er kann bestenfalls noch und sollte auch in Übungen und Tutorien hergestellt werden.

Dabei muss berücksichtigt werden, dass die Studierenden, wenn sie zur Universität kommen, in aller Regel den persönlichen Kontakt mit den Lehrenden wünschen. Ansonsten könnte man entsprechend dem Vorgehen der Fernuniversitäten alles im Fernstudium erledigen, womit der Universität auf dieser Stufe nur noch die Durchführung der Prüfungen als Aufgabe verbliebe. Die eigentlichen Bereiche, in welchen universitäre Lehre angesiedelt wäre, wären dann das Master- und das Ph.D.-Studium. Ein solches Modell ist denkbar; ob es anzustreben ist, mag an dieser Stelle offen bleiben.

Wie immer man die Bachelorstufe ausgestalten will, auf der Masterstufe können die elektronischen Medien nicht die gleiche Funktion haben. Hier sollte in kleineren Gruppen unterrichtet werden, die einen persönlichen Kontakt erlauben. Dies ist heute bei weitem nicht überall der Fall: Jahrgänge mit 150 Anfängern z.B. im Bereich Banking and Finance, wie wir sie in St. Gallen erleben, reproduzieren auf der Masterstufe das Massenproblem der Bachelorstufe, wenn auch in etwas geringerem Ausmaß. Verbesserungen sollten neben dem erforderlichen Ausbau des Lehrkörpers jedoch auch dadurch möglich werden, dass es zum einen durch den schwierigeren Zugang auf dieser Stufe weniger Studierende gibt und dass zum anderen auf der Bachelorstufe durch den oben diskutierten Einsatz elektronischer Medien Lehrkapazität frei wird.

Im Zuge der Internationalisierung wird auf der Masterstufe zunehmend ausschließlich in Englisch unterrichtet werden. Damit kann man Studierende anziehen, deren Muttersprache nicht Deutsch ist, womit man sich aber auch in den internationalen Wettbewerb stellt. Dies wird vor allem dann relevant werden, wenn mit höheren Studiengebühren auf dieser Stufe der heutige Wettbewerbsvorteil (sehr) geringer Studiengebühren wegfallen sollte. Dies gilt auch, wenn ein funktionierendes Stipendiensystem eingerichtet sein sollte. 


\section{Gebhard Kirchgässner}

In wieweit sich grundlegende Änderungen beim Doktorat bzw. Ph.D. ergeben werden, bleibt abzuwarten, sieht man einmal davon ab, dass hier die Lehre fast ausschließlich nur noch in Englisch stattfinden dürfte. ${ }^{31}$ Auch wenn wir heute unser (traditionelles) Doktorat gerne mit dem angelsächsischen Ph.D. gleichsetzen, ist doch nicht zu übersehen, dass zwischen beiden Konzepten Welten liegen. Der angelsächsische Ph.D. ist im Allgemeinen eine Qualifikation zum Eintritt in die Wissenschaft, während die Mehrheit unserer Doktorierenden nach Abschluss der Promotion eine Tätigkeit außerhalb des akademischen Bereichs aufnimmt. Dies gilt auch für die Volkswirtschaftslehre, in besonderem Maße aber für die Betriebswirtschaftslehre, und es gilt auch dann, wenn wir die Definition des akademischen Bereichs weit fassen. Dies bedeutet nicht, dass nicht auch unter unseren Doktorierenden hervorragender wissenschaftlicher Nachwuchs ist, aber die Zahl derer, die eine Karriere in der Wissenschaft anstreben, ist eindeutig in der Minderheit; die Mehrheit strebt eine Tätigkeit außerhalb der universitären Institutionen an. Angesichts der hohen Zahl der abgeschlossenen Promotionen ist dies auch gar nicht anders möglich; diese Zahl ist weit höher als das, was im Verhältnis zum akademischen Personal an guten amerikanischen oder britischen Universitäten heute üblich ist.

Einige Universitäten sind formal bereits weitgehend auf das angelsächsische Modell eingeschwenkt. Sie verlangen zum einen im Rahmen ihres Ph.D.Programms ein nochmals ein bis zwei Jahre dauerndes Studium, an dessen Ende eine Prüfung steht, die bestanden werden muss, bevor man zur Dissertation zugelassen werden kann. Zweitens erfolgt die Promotion kumulativ: Die schriftliche Promotionsleistung besteht aus „3 Papers on ...“. Ist die Kursphase anspruchsvoll und verlangt man zudem tatsächlich, wie es zumindest in Promotionsordnungen steht, dass diese Papiere eine so hohe Qualität aufweisen, dass sie in guten internationalen Zeitschriften veröffentlicht werden können, dürfte sich die Zahl der Promotionen deutlich reduzieren. Damit verschwindet freilich nicht die Nachfrage nach der traditionellen Promotion. Will man diese als Alternative beibehalten, dürfte es explizit oder implizit eine Aufspaltung geben: in den stärker wissenschaftlich orientierten Ph.D., der dann auch in aller Regel eine notwendige, wenn auch nicht hinreichende Vorbedingung für eine wissenschaftliche Laufbahn sein wird, und eine mehr praktisch ausgerichtete Promotion, welche diejenigen anstreben, die ihre berufliche Zukunft außerhalb des akademischen Bereichs sehen. Ob dies auch im verliehenen Titel zum Ausdruck gebracht werden wird, dem Ph.D. und dem Dr., ist fraglich. Man mag dies als sinnvoll erachten, aber es wird einen erheblichen Druck geben, dass allen der Titel Ph.D. verliehen wird. Insbesondere auch jene Betriebswirte, die selbst keine wissenschaftliche Laufbahn anstreben, werden ihn für sich beanspruchen.

\section{Die wissenschaftliche Forschung}

Die zu Beginn bestehende Konzentration auf die Lehre hatte und hat bis heute zur Konsequenz, dass im Vergleich zu den normalen' Universitäten die Lehre ein größeres Gewicht hatte und die Forschung teilweise eher ein Mauerblümchendasein führte. Dies gilt für St. Gallen und Wien, und in Mannheim mindestens für 
die betriebswirtschaftliche Fakultät. Es gilt - zumindest im deutschsprachigen Raum - aber auch für die betriebswirtschaftlichen Fakultäten an normalen Universitäten, und seien sie auch so berühmt wie z.B. Köln, wie sich noch vor 10 Jahren an internationalen Rankings leicht zeigen ließ ${ }^{32}$ Dies galt sicher weniger für die volkswirtschaftlichen Bereiche der Wirtschaftsuniversitäten und noch weniger für die volkswirtschaftlichen Fakultäten zumindest einiger ,normaler' Universitäten wie z.B. Bonn oder Wien, aber von den drei Wirtschaftsuniversitäten im deutschsprachigen Raum hat bis vor etwa 10 Jahren nur Mannheim mit hervorragenden Forschungsleistungen auf sich aufmerksam gemacht; noch in den neunziger Jahren galt die ,Journalforschung' auch an meiner eigenen Universität nicht sehr viel; sie wurde eher geduldet als gefördert. Dies hat sich in den Forschungsrankings deutlich niedergeschlagen. Im Forschungsranking für die volkswirtschaftlichen Fakultäten im deutschsprachigen Raum für die Jahre 1990 bis 1995 kam zwar Mannheim auf Platz 3, St. Gallen jedoch nur auf Platz 27 und die Wirtschaftsuniversität Wien lag abgeschlagen auf dem 65. von insgesamt 72 Plätzen. ${ }^{33}$

Dies hat sich in den vergangenen zehn Jahren massiv geändert. Im HandelsblattRanking des Jahres 2009 der volkswirtschaftlichen Fakultäten im deutschsprachigen Raum nimmt Mannheim auf Platz 2 (hinter Zürich und noch vor Bonn) nach wie vor eine Spitzenposition ein, St. Gallen ist auf Platz 8 vorgerückt, und Wien liegt immerhin auf Platz 20. ${ }^{34}$ Offensichtlich haben St. Gallen und Wien in dieser Hinsicht mit Erfolg erhebliche Anstrengungen unternommen.

Was die Betriebswirtschaftslehre angeht, liegen die Wirtschaftsuniversitäten Wien, St. Gallen und Mannheim zwar im entsprechenden Handelsblatt-Ranking, welches im Jahr 2009 zum ersten Mal erschien, (in dieser Reihenfolge) auf den ersten drei Plätzen, ${ }^{35}$ aber dies darf nicht darüber hinwegtäuschen, dass sie international betrachtet nach wie vor kaum erstklassig sind. Im Financial Times Ranking der European Business Schools des Jahres 2010 liegt St. Gallen auf Platz 16, Mannheim auf Platz 32 und die Wirtschaftsuniversität Wien abgeschlagen auf Platz 51 unter insgesamt 75 bewerteten Institutionen. ${ }^{36}$ Diese vergleichsweise schlechten Platzierungen, die zeigen, dass es für diese Universitäten noch ein weiter Weg sein wird, bevor die in ihren Leitbildern bzw. Visionen angestrebten Ziele erreicht sein werden, sind nicht weiter verwunderlich, da der notwendige Kulturwandel in diesem Bereich nicht von heute auf morgen vollzogen werden kann; er stellt (auch) ein Generationenproblem dar, und die durchschnittliche Amtsdauer von

32. In einem Ranking der europäischen Business Schools aus dem Jahr 2000 ist keine der drei Wirtschaftsuniversitäten unter den 45 besten aufgeführt, wohl aber das IMD in Lausanne. Siehe Baden-Fuller, Ravazzolo und Schweizer (2000). In einem Ranking von Baden-Fuller aus dem Jahr 2001 liegen Mannheim an 32. und St. Gallen an 74. Stelle; die Wirtschaftsuniversität Wien ist unter den 104 ,Schulen' gar nicht aufgeführt. Von den ,normalen' Universitäten sind nur Kiel (Platz 44), Mainz (Platz 78), Wien und Bonn sowie das WZB Berlin (alle Platz 96) aufgeführt. Siehe http://www.mittelbau.unisg.ch/org/mittelbau/ web.nsf/ c2d5250e0954edd3c12568e40027f306/fbe169fce70b62f0c1256c75002ab6e1/\$FILE/List_top_100. pdf $(07 / 12 / 10)$.

33. Siehe hierzu Bommer und Ursprung (1998, S. 14f.).

34. Siehe http://www.handelsblatt.com/_t $=$ dgtool,id=16,obj=1;singleclip/ $(05 / 12 / 10)$.

35. Siehe http://www.handelsblatt.com/bwl-unis/ (05/12/10).

36. Siehe http://rankings.ft.com/exportranking/european-business-school-rankings-2010/pdf $(24 / 01 / 11)$. 


\section{Gebhard Kirchgässner}

Hochschullehrern dürfte zwischen 20 und 30 Jahren liegen. Der Wandel wird dadurch jedoch erleichtert, dass zunehmend auch Professoren aus dem nichtdeutschsprachigen Ausland berufen werden. ${ }^{37}$

Damit stellt sich die Frage, weshalb es überhaupt zu diesem Wandel gekommen ist. Hier spielen die viel gescholtene Bologna Reform bzw. die mit ihr verbundenen Akkreditierungen eine wesentliche Rolle. Bei der Akkreditierung durch EQUIS bzw. AACSP, ${ }^{38}$ die beiden international führenden Akkreditierungsorganisationen, bei denen eine ,Business School' heute akkreditiert sein muss, wenn sie international mithalten will, kamen neben Vertretern der Wirtschaft auch Kollegen aus dem angelsächsischen Raum als Gutachter nach St. Gallen, die ein Verständnis von Forschung hatten, welches sich in seinen Spielregeln stark an der naturwissenschaftlichen Forschung orientiert: Die Scientific Community ist international und nicht auf einen kulturellen oder sprachlichen Raum begrenzt, und der Qualitätsausweis wissenschaftlichen Arbeitens besteht im Wesentlichen darin, die eigenen Arbeiten auf internationalen Konferenzen vorzutragen sowie in hoch angesehenen internationalen Zeitschriften zu publizieren.

Man kann sich selbstverständlich fragen, weshalb sich diese Art der Forschungskultur in den Wirtschaftswissenschaften in den deutschsprachigen Ländern erst so spät durchsetzen konnte. Dabei ist zunächst zwischen der Volks- und der Betriebswirtschaftslehre zu unterscheiden. Die deutschsprachige Volkswirtschaftslehre hatte bis zur Zeit des Nationalsozialismus internationales Format, wobei sich Österreich besonders hervorgetan hat, um nur Josef A. Schumpeter und Friedrich August von Hayek als zwei der hervorragenden Beispiele zu nennen. ${ }^{39}$ Sie wurde jedoch - ähnlich wie die Naturwissenschaften - durch den Zweiten Weltkrieg international weitgehend abgekoppelt. Dies geschah auch dadurch, dass hervorragende Ökonomen ausgewandert sind. Sie hat danach - anders als die Naturwissenschaften - den internationalen Anschluss erst spät wieder gefunden. Dabei spielte auch eine wesentliche Rolle, dass durch den Zweiten Weltkrieg Deutsch als Wissenschaftssprache international verschwunden und vom Englischen vollständig verdrängt worden ist. Dies war davor anders: Deutschsprachige Lehrbücher wurden z.B. in Skandinavien vor dem Zweiten Weltkrieg als Standardliteratur verwendet. Dadurch, dass von den deutschsprachigen Ökonomen nach dem Zweiten Weltkrieg nach wie vor im Wesentlichen auf Deutsch publiziert wurde, hat man sich weitgehend selbst isoliert. Es benötigte viel, diese Isolation zu durchbrechen. Voraussetzung dafür war, dass man sich zunächst einmal dessen bewusst wurde, dass ein Problem darin bestand, dass die deutsche Volkswirtschaftslehre international nicht präsent war. Um dies zu ändern, wurde z.B. unter Hans-Werner Sinn als Vorsitzendem des Vereins für Socialpolitik, der Vereinigung der deutschsprachigen Ökonomen, die Zeitschrift für Wirtschafts- und Sozialwissenschaften, das altehrwürdige Schmollers

37. Die Ausrichtung auf solche Rankings, seien es Publikationen oder Zitationen, die gezählt werden, ist freilich nicht unumstritten. (Siehe hierzu z.B. Frey und Rost (2008) oder Osterloh und Frey (2009).) Andererseits kann man sich diesem Wettbewerb heute nicht entziehen. Dies gilt insbesondere dann, wenn man im Markt der Executive Education tätig werden will, worauf unten noch eingegangen wird.

38. European Quality Improvement System bzw. Association to Advance Collegiate Business Schools.

39. In der Schweiz haben die bedeutendsten Ökonomen, Vilfredo Pareto und Léon Walras, freilich in Lausanne und damit im französischen Sprachraum gelehrt. 


\section{Kaderschmieden der Wirtschaft und/oder Universitäten?}

Jahrbuch, welches im Jahr $2010 \mathrm{im}$ 130. Jahr erschienen ist, im Jahr 2000 durch das German Economic Review als Zeitschrift der Gesellschaft ersetzt. In Schmollers Jahrbuch konnte man auch in Englisch veröffentlichen; die Masse der Beiträge war aber in Deutsch. Im German Economic Review ist Englisch Pflicht. ${ }^{40}$ Empirica, die Zeitschrift der Nationalökonomischen Gesellschaft Österreichs, veröffentlicht seit längerem nur noch auf Englisch. Und seit 2007 publiziert auch die seit dem Jahr 1865 erscheinende Schweizerische Zeitschrift für Volkswirtschaft und Statistik, in der früher immer auf Deutsch und Französisch publiziert werden konnte, als Swiss Journal of Economics and Statistics nur noch auf Englisch.

Hans-Werner Sinn hat darüber hinaus im Jahr 1997 die drei Nationalbanken bewogen, Geld für ,Vortragsprämien' bereitzustellen. Der Verein stellte eine Liste führender internationaler Konferenzen auf, bei denen Referatsangebote einem Zulassungsverfahren unterworfen werden, und wer als junge Ökonomin oder junger Ökonom (unter 30 Jahren) es schafft, auf einer dieser Konferenzen vorzutragen, bekommt einen Zuschuss in Höhe von 500 Euro (bzw. früher 1000 DM). Dabei ist es zum Teil sehr schwierig, dort vortragen zu dürfen; die Ablehnungsquoten liegen teilweise über 50 Prozent, und junge, noch unbekannte Bewerber haben es in einem solchen Verfahren naturgemäß schwerer als jene, deren Namen in der Scientific Community bereits etwas gilt.

Heute ist die Volkswirtschaftslehre in den deutschsprachigen Ländern international angekommen, sie hat aber insbesondere gegenüber unseren kleineren europäischen Nachbarn immer noch einen Rückstand. Die Tatsache, dass die skandinavischen Länder oder die Niederlande zu klein für einen wissenschaftlichen Markt in ihrer Landessprache waren, hat dazu geführt, dass Englisch bei ihnen schon sehr früh die Sprache ihrer (wissenschaftlichen) Publikationen wurde, was auch dadurch gefördert wurde, dass in der Volkswirtschaftslehre grundsätzlich auf Englisch unterrichtet wird. Der Vorsprung, welchen sie dadurch haben, wird z.B. dadurch deutlich, dass das Scandinavian Journal of Economics in den internationalen Ranglisten vergleichsweise hoch rangiert, während das German Economic Review, falls es überhaupt gelistet ist, unter ,ferner liefen' angesiedelt ist. ${ }^{41}$

Die Betriebswirtschaftslehre konnte als vergleichsweise junge Wissenschaft nie jenes internationale Ansehen haben, welches die deutschsprachige Volkswirtschaftslehre vor dem Zweiten Weltkrieg hatte. Hier gab es freilich auch weniger Anreize, sich in den internationalen Wissenschaftsbetrieb einzuklinken. Abgesehen davon, dass diskutiert wurde, ob die Betriebswirtschaftslehre überhaupt eine Wissenschaft (im Sinne der Sciences) und nicht vielmehr eine Kunst ist, weshalb es im akademischen Unterricht in erster Linie darum geht, die Kunst guter Unternehmensführung zu lehren, ${ }^{42}$ hat man Forschung im Wesentlichen als , angewandte Forschung' im Sinne der Unterstützung bei der Lösung betrieblicher Probleme begriffen, die dann in Zusammenarbeit mit dem jeweiligen Unternehmen

40. Daneben gibt es noch die Perspektiven der Wirtschaftspolitik, in denen politikorientierte Arbeiten auf Deutsch publiziert werden können. Sie spielen im Selbstverständnis des Vereins für Socialpolitik sowie in seiner Außendarstellung freilich eine untergeordnete Rolle.

41. Im Handelsblatt Zeitschriften Ranking 2010 belegt das Scandinavian Journal of Economics mit einem Gewicht von 0.2 (zusammen mit 76 anderen Zeitschriften den Rang 83, während das German Economic Review mit einem Gewicht von 0.1 (zusammen mir 166 weiteren Zeitschriften) nur auf Platz 271 liegt. Siehe http://www.handelsblatt.com/singleclip.aspx?_t=dgtool\&id=15\&obj=1 (05/12/10).

42. Siehe hierzu z.B. http://www.chrmglobal.com/Articles/180/1/Management-Art-or-Science-.html $(07 / 12 / 10)$. 


\section{Gebhard Kirchgässner}

durchgeführt wird. Dies hatte zwei Vorteile: Zum einen entzog man sich damit dem (internationalen) wissenschaftlichen Wettbewerb, zum anderen ließen sich damit in erheblichem Maße Drittmittel einwerben, sei es zur Anstellung weiterer Mitarbeiter, sei es zur Aufbesserung des eigenen Gehalts. Und selbst dort, wo man eigene theoretische Ideen entwickelte, blieb dies international weitestgehend bedeutungslos. So ist z.B. das von Hans Ulrich entwickelte St. Galler Modell der Managementlehre, welches von vielen jener Kollegen, die in St. Gallen wissenschaftlich sozialisiert wurden, immer noch hochgehalten wird und mit dem sich auch noch gutes Geld verdienen lässt, außerhalb von St. Gallen weitgehend und außerhalb des deutschen Sprachraums völlig unbekannt. ${ }^{43}$

Für viele Kolleginnen und Kollegen aus dem Bereich der Betriebswirtschaftslehre war es daher ein Schock, als bei den Akkreditierungen verlangt wurde, dass sie in Zukunft ,Journalforschung' machen. Bei einem Teil der Professorenschaft stieß dies auf erhebliche Ablehnung. Zwar gibt es einige Fakultäten, die vergleichsweise strikt eine internationale Orientierung in der Forschung anstreben, aber es wird doch einige Zeit (und vermutlich länger als im Bereich der Volkswirtschaftslehre) dauern, bis ein entsprechender Wandel allgemein vollzogen ist.

\section{Die Beratung}

Die so bezeichnete, angewandte Forschung', d.h. die Unterstützung von Betrieben bei der Lösung ihrer Probleme, ist eher als Beratung denn als wissenschaftliche Forschung zu charakterisieren. Dennoch ist zu berücksichtigen, dass auch sie zum Aufgabenbereich der Wirtschaftsfakultäten bzw. -universitäten gehört. Deren Aufgabengebiet geht traditionell über den Bereich von Forschung und Lehre (und Selbstverwaltung) hinaus, es umfasst auch die Beratung, sei es jene von Betrieben im Rahmen der Betriebswirtschaftslehre oder jene öffentlicher Institutionen im Rahmen der Volkswirtschaftslehre. So sind z.B. die Wissenschaftliche Beiräte der Wirtschafts- und Finanzministerien oder entsprechende Sachverständigenräte ohne die Mitwirkung von Wirtschaftsprofessoren kaum denkbar (und wären auch ohne sie kaum sinnvoll). Das Problem ist somit nicht ein entweder oder, sondern ein sowohl als auch. Freilich ging es traditionell insbesondere im Bereich der Betriebswirtschaftslehre sehr einseitig in eine Richtung, und dies wird sich ändern müssen.

In dieser Praxisorientierung ähneln die Wirtschaftswissenschaftler den Medizinern wie den Technikern: Es ist kaum vorstellbar, dass ein Medizinprofessor nicht auch selbst Patienten betreut, und Techniker entwickeln, zum Teil in Zusammenarbeit mit privaten Firmen, auch Produkte, die kommerziell verwertbar sind. Für Betriebswirte, kaum für Volkswirte, gilt dabei genauso wie für Mediziner und Techniker (und auch für manche Juristen), dass das dabei legal erzielte ,Nebeneinkommen' höher als das offizielle Gehalt sein kann, welches man als voll bezahlter Universitätsprofessor erhält. Es ist in diesem Bereich nicht selten, dass

43. Zum St. Galler Modell der Managementlehre siehe z.B. Ulrich und Krieg (1972) sowie Dubs et al. (2004). Fredmund Malik, Schüler von Hans Ulrich und Titularprofessor an der Universität St. Gallen, hat mit seinem Management Zentrum St. Gallen ein florierendes, international tätiges Unternehmen mit weltweit 300 Mitarbeitern geschaffen. Für das dort vermittelte Wissen stellt dieses Modell nach wie vor den theoretischen Kern dar. 
Professoren neben ihrer universitären Tätigkeit ganz offiziell auch in ihrer eigenen Firma (bzw. Kanzlei) tätig sind.

Diese praktischen Tätigkeiten haben zwei Seiten. Zum einen ist es gerade in Fächern wie der Betriebswirtschaftslehre (und der Rechtswissenschaft) sinnvoll, dass akademische Lehrer auch praktische Erfahrung haben. Dies kann zum Teil, aber nicht durchgehend dadurch bewerkstelligt werden, dass Lehrbeauftragte unterrichten. Zum anderen aber stehen diese praktischen Tätigkeiten in Konkurrenz zur eigentlichen wissenschaftlichen Forschung. Dies gilt auch dort, wo die praktische Tätigkeit als, angewandte Forschung' bezeichnet wird.

Soweit ein bestimmter Rahmen nicht überschritten wird, wird insbesondere dann, wenn die eingeworbenen Gelder nicht in private Taschen fließen, von der offiziellen Politik derartig unternehmerisches Verhalten heute gefordert und gefördert: Die (erwartete) Einwerbung von Drittmitteln gilt als Qualitätsausweis, und sie kann bei Berufungsverhandlungen eine Rolle spielen. Auch hier ist die Angelegenheit wieder ambivalent. Zum einen kann man damit durch die Anstellung zusätzlicher Mitarbeiterinnen und Mitarbeiter den Bereich der Forschung ausdehnen, zum anderen wird der Lehrstuhlinhaber, der - davon sollte man zumindest in aller Regel ausgehen können - auf Grund eigener hervorragender Forschung in sein Amt berufen wurde, zunehmend zum Forschungsmanager. Dazu ist er möglicherweise wenig geeignet; vor allem aber fehlt ihm dann die Zeit, selbst Forschung zu betreiben. Dies gilt auch dann, wenn es sich um Mittel für die Grundlagenforschung handelt, die von den nationalen Forschungseinrichtungen vergeben werden.

Es gibt somit nicht nur das Konkurrenzverhältnis zwischen Lehre und Forschung bezüglich der Zeitverwendung, ${ }^{44}$ sondern, wenn man die Verpflichtungen in der Lehre einmal als gegeben ansieht, das noch viel bedeutendere Konkurrenzverhältnis zwischen Forschung und Beratung. Im Bereich der Volkswirtschaftslehre sind die Möglichkeiten, mit Hilfe von Gutachten Drittmittel einzuwerben, im Allgemeinen freilich sehr viel geringer als bei den Betriebswirten. Dies ändert nichts daran, dass die Arbeit an solchen Gutachten, so nützlich sie auch sein mag, häufig von wissenschaftlicher Forschung im engeren Sinn weit entfernt ist. Auch hier stellt sich das Problem, dass die Zeit nur einmal verwendet werden kann.

Dieses Konkurrenzverhältnis in der Verwendung der Zeit wird dann besonders relevant, wenn man neben der Beratungstätigkeit in der internationalen Forschung präsent sein will. Es kann zwar sehr befriedigend sein, ist aber alles andere als einfach, gleichzeitig ein hohes Ansehen in der (internationalen) Wissenschaftlergemeinschaft $\mathrm{zu}$ haben und in der Politikberatung tätig zu sein. Außerdem ist nicht jede ökonomische Forschung, auch wenn sie sich mit politisch relevanten Fragen befasst, wirtschaftspolitisch einfach umsetzbar.

Dieser Konflikt spielt sich nicht nur innerhalb der einzelnen Wissenschaftler ab, sondern auf höherer Ebene auch in den Fakultäten. Sie müssen sich entscheiden, wie sie ihre Forschung ausrichten wollen. Man kann theoretische Forschung betreiben, die intellektuell äußerst befriedigend ist und die auch Grundlagen für spätere stärker angewandte Forschungen bietet, die aber in keiner Weise zur Politikberatung befähigt. Es gibt aber auch angewandte Forschung, die vergleichsweise direkt anwendbar ist. Dies gilt insbesondere für empirische Arbeiten, in denen die 


\section{Gebhard Kirchgässner}

Wirkungen wirtschaftspolitischer Instrumente wie z.B. der Geldpolitik oder der aktiven Arbeitsmarktpolitik untersucht werden. Nicht umsonst halten sich z.B. Zentralbanken größere Forschungsabteilungen, und einzelne der dort forschenden Ökonomen sind voll in die internationale Wissenschaftlergemeinschaft integriert. Für Wirtschaftsuniversitäten, deren Hauptaufgabe in der Lehre - wie mehrfach erwähnt - die Ausbildung von Betriebswirten ist, dürfte es sinnvoll sein, auch die volkswirtschaftliche Forschung stärker angewandt, d.h. auf konkrete wirtschaftspolitische Fragestellungen, auszurichten. ${ }^{45}$

\section{Die Weiterbildung}

Neben der universitären Ausbildung wird die Weiterbildung in Zukunft vermutlich eine sehr viel stärkere Rolle an den Wirtschaftsuniversitäten spielen als heute, sieht man einmal von St. Gallen ab, welches im deutschsprachigen Raum in diesem Bereich eine Vorreiterrolle einnimmt. Da in diesem Bereich hohe Studiengebühren verlangt werden können und auch verlangt werden, ergibt sich hier in Zeiten knapper Staatskassen eine zusätzliche Finanzierungsquelle. Zwar ist die Metapher von der Universität als Unternehmen für den akademischen Bereich fehl am Platz, zumindest solange man noch das Ideal einer Gemeinschaft von Lehrenden und Lernenden im Hinterkopf hat, da Studierende keine Kunden sind und auch nicht als solche betrachtet werden sollten. Im Bereich der Weiterbildung ist die Universität dagegen ein Unternehmen, welches sich zudem in einen internationalen Wettbewerb begibt, welcher noch viel härter als jener im rein akademischen Bereich ist. So bestimmen z.B. Rankings wie dasjenige der Financial Times mit darüber, wie hoch die Gebühren sind, die man für die entsprechenden MBA bzw. EMBA Programme verlangen kann. In diese Rankings geht neben der Forschungsleistung einer Universität auch die Zufriedenheit der Teilnehmer dieser Lehrgänge ein. Eine derartige ,Executive School' muss daher professionell geführt werden; unsere traditionellen akademischen Strukturen dürften kaum dafür geeignet sein. Es bietet sich daher an, sie zumindest weitgehend aus dem normalen Universitätsbetrieb auszugliedern.

Damit stellt sich die Frage, welche Rolle in diesem Bereich die eigentliche (akademische) Universität spielen soll. Selbstverständlich gibt sie dafür ihren Namen, womit sie auch eine Kontrollfunktion übernehmen muss, um sicherzustellen, dass ihre Reputation nicht gefährdet wird. ${ }^{46}$ Eine andere Frage ist, inwieweit sich der Lehrkörper der Universität dort engagieren soll. Auch hier stellt sich wieder die Konkurrenz in der Zeitverwendung. Angesichts der Tatsache, dass mit diesen Programmen viel Geld verdient werden kann, gibt es jedoch auch die Möglichkeit, dass Dozenten der Universität dort einen Teil ihres Lehrdeputats ableisten und mit dadurch zusätzlich eingenommenem Geld weitere Professuren eingerichtet werden können.

45. Zum Verhältnis zwischen Theorie und Empirie in der wirtschaftswissenschaftlichen Forschung siehe auch Morgan (1995).

46. Ohne dass sie sich dagegen wehren kann, werden auch andere versuchen, von dieser Reputation zu profitieren. Dies gilt in St. Gallen z.B. für das Management Zentrum St. Gallen oder die Management School St. Gallen, die beide nichts mit der Universität St. Gallen zu tun haben, was aber für Außenstehende nicht ohne weiteres ersichtlich ist. 


\section{Kaderschmieden der Wirtschaft und/oder Universitäten?}

\section{Abschließende Bemerkungen}

Neben der akademischen Selbstverwaltung, die immer dazu gedacht werden muss, gibt es somit vier Bereiche, in denen Wirtschaftsuniversitäten (und teilweise auch Wirtschaftsfakultäten) tätig sind, Lehre, akademische Forschung, Beratung sowie Weiterbildung, und in allen vier Bereichen stehen sie vor erheblichen Herausforderungen. In Zusammenhang mit der Finanz- und Wirtschaftskrise der vergangenen Jahre haben sich für die Wirtschaftswissenschaften aber noch ganz andere Herausforderungen ergeben. Insbesondere im deutschsprachigen Raum wurde die moderne Ausrichtung der Volkswirtschaftslehre (gemäß angelsächsischem Vorbild) mit ihrer Mathematisierung kritisiert und eine Rückbesinnung auf die eher deutsche Tradition der Ordnungstheorie im Sinne z.B. der Freiburger Schule von Walter Eucken gefordert. Abgesehen davon, dass die Anliegen der alten Ordnungstheorie in der modernen Institutionenökonomik aufgenommen wurden und dass empirische Untersuchungen, die der Wirtschaftspolitik dienlich sein können, kaum ohne entsprechende Mathematik möglich sind, dürfte ein deutscher Sonderweg und damit eine Abkoppelung von der internationalen Entwicklung wenig Erfolg versprechend sein. ${ }^{47}$ Damit soll nicht bestritten werden, dass, wie oben bereits ausgeführt wurde, ein Teil der modernen ökonomischen Theorie (zumindest prima facie) kaum erkennen lässt, dass mit seiner Hilfe wirtschaftspolitische Probleme besser bewältigt werden können. ${ }^{48}$

Während der Volkswirtschaftslehre in diesem Zusammenhang eine eher indirekte Mitschuld an der Krise vorgeworfen wurde, da sie die Wirtschaftskrise nach dem Eintreten der Finanzkrise nicht kommen sah, was z.B. durch die sehr positiven Wachstumsprognosen für das Jahr 2009 belegt werden kann, die noch im Frühjahr und Sommer 2008 von wichtigen Institutionen wie z.B. den deutschen Wirtschaftsforschungsinstituten oder dem Weltwährungsfonds erstellt wurden, wird der Betriebswirtschaftslehre der Vorwurf einer sehr viel direkteren Schuld an der Krise gemacht: Durch die Betonung des Gewinnmotivs und der Rechte der Anteilseigner eines Unternehmens im Rahmen des Konzeptes des Shareholder-Values soll sie jene Manager gleichsam herangezüchtet haben, die durch ihre Gier die Krise verursacht haben. ${ }^{49}$ Hier liegt zwar eine sehr verkürzte Vorstellung der akademischen Ausbildung im Bereich der Betriebswirtschaftslehre zugrunde, aber zumindest in Experimenten hat sich gezeigt, dass Studierende der Wirtschaftswissenschaften im Allgemeinen stärker eigennutzorientiert handeln als Studierende anderer Fakultäten. Dabei ist freilich nach wie vor offen, inwieweit dies eine Frage der Ausbildung und inwieweit eine Frage der Selbstselektion ist: Sind Studierende der Betriebswirtschaftslehre schon zu Beginn ihres Studiums stärker eigennützig und/oder materiell orientiert als Studierende anderer Fächer? ${ }^{50}$ Vermutlich spielt beides eine Rolle. Aber selbst wenn alles nur Selbstselektion wäre, muss man sich mit der Forderung auseinandersetzen, dass die akademische

47. Siehe hierzu Kirchgässner (2009).

48. Siehe hierzu Kirchgässner (2011).

49. Siehe z.B. Knellwolf, Sind HSG-Studenten ,falsch programmierte' Roboter?. Tagesanzeiger vom 8. April 2009 (http://www.tagesanzeiger.ch/schweiz/standard/Sind-HSGStudenten-falschprogrammierte-Roboter/ story/25264066 (07/12/10)). Siehe hierzu auch Kirchgässner (2009).

50. Siehe hierzu Kirchgässner (2005). 


\section{Gebhard Kirchgässner}

Ausbildung in den Wirtschaftswissenschaften auch ethische Werte vermitteln sollte, und zwar auch solche, die über die wissenschaftsimmanenten Werte wie z.B. Korrektheit im Umgang mit dem intellektuellen Eigentum anderer hinausgehen.

Die Universität Harvard lässt in diesem Zusammenhang von ihren MBAAbsolventen auf freiwilliger Basis einen Eid ablegen, welcher dem hippokratischen Eid der Ärzte nachgebildet wurde und mit welchem diese versprechen, als Manager in Zukunft die Interessen der Aktionäre, Mitarbeiter und Kunden ihres Unternehmens sowie der Gesellschaft, in der sie leben, zu gewährleisten. ${ }^{51}$ Abgesehen davon, dass dies in keiner Weise einklagbar ist, ist es auch sehr fraglich, ob es irgendetwas bewirkt. Solange man nicht offensichtlich kriminell wird, werden intelligente Leute (fast) immer im Nachhinein eine Begründung dafür finden, weshalb eine bestimmte wirtschaftliche Handlung aus ihrer Perspektive gerechtfertigt war, auch wenn sie großen Schaden angerichtet hat, und sei es auch nur, dass sie sich auf ihre gute Absicht und die Fehlerhaftigkeit allen menschlichen Handelns berufen. ${ }^{52}$ Zudem darf man fragen, woher sich eine Institution in einem pluralistischen Staat das Recht nehmen darf, erwachsenen Menschen bestimmte Werthaltungen vorschreiben zu wollen; auch Verfassung und Gesetze können keine Gesinnung verlangen: Sie verlangen ausschließlich, dass man sich an bestimmte Regeln hält.

Andererseits stellt sich die Frage, inwieweit die Studien der Volks- und der Betriebswirtschaftslehre über rein wirtschaftswissenschaftliche Fragestellungen hinausgehen sollen. Es ist sicher sinnvoll, den Studierenden die Möglichkeit zu geben, auch Lehrveranstaltungen aus anderen Sozialwissenschaften sowie auch aus den Geisteswissenschaften zu besuchen, die sie auf ihre erforderlichen Kreditpunkte anrechnen lassen können. Dazu sollten auch Angebote in Philosophie und in Ethik (Wirtschaftsethik) gehören. Inwieweit man dies jedoch vorschreiben sollte, ist eine offene Frage. St. Gallen hat den Besuch solcher Veranstaltungen sowohl auf der Bachelor- als auch auf der Masterstufe vorgeschrieben; sie machen 25 Prozent des gesamten Lehrprogramms aus. Von vielen Studierenden werden sie jedoch gerade auf der Masterstufe eher als lästige Pflicht empfunden, derer man sich

51. Die Formel lautet: "As a manager, my purpose is to serve the greater good by bringing people and resources together to create value that no single individual can create alone. Therefore I will seek a course that enhances the value my enterprise can create for society over the long term. I recognize my decisions can have far-reaching consequences that affect the well-being of individuals inside and outside my enterprise, today and in the future. As I reconcile the interests of different constituencies, I will face choices that are not easy for me and others.

Therefore I promise:

I will act with utmost integrity and pursue my work in an ethical manner.

I will safeguard the interests of my shareholders, co-workers, customers and the society in which we operate. I will manage my enterprise in good faith, guarding against decisions and behavior that advance my own narrow ambitions but harm the enterprise and the societies it serves.

I will understand and uphold, both in letter and in spirit, the laws and contracts governing my own conduct and that of my enterprise.

I will take responsibility for my actions, and I will represent the performance and risks of my enterprise accurately and honestly.

I will develop both myself and other managers under my supervision so that the profession continues to grow and contribute to the well-being of society.

I will strive to create sustainable economic, social, and environmental prosperity worldwide.

I will be accountable to my peers and they will be accountable to me for living by this oath.

This oath I make freely, and upon my honor."

52. Siehe hierzu z.B. Vaubel (2010). 
mit möglichst geringem Aufwand zu entledigen sucht. Die Hoffnung, dass durch das Vorschreiben solcher Pflichtveranstaltungen die späteren Entscheidungen der heute Studierenden, wenn sie einmal im Berufsleben stehen werden, in die, richtige Richtung' beeinflusst werden, kann man auch als naiv betrachten.

Insgesamt stehen die Wirtschaftswissenschaften und damit die entsprechenden Universitäten und Fakultäten vor erheblichen Herausforderungen, wenn sie ihrem gesellschaftlichen Auftrag in diesem Jahrhundert gerecht werden wollen. Es spricht aber nichts dagegen, dass sie diese Herausforderungen auch meistern werden. Andererseits sollte man mit Prognosen über die zukünftige Entwicklung sehr vorsichtig sein, insbesondere wenn man ein ganzes Jahrhundert als Horizont anvisiert. Die Probleme, die hier aufgezeigt wurden, fordern uns heute und in der näheren Zukunft; bis in 20 Jahren kann sich die Situation deutlich geändert haben. ${ }^{53}$ Dies entbindet uns freilich nicht von der Aufgabe, die heute erkannten Probleme anzugehen.

\section{Literaturverzeichnis}

Baden-Fuller, C., F. Ravazzolo und T. Schweizer (2000), Making and Measuring reputations: The Research Ranking of European Business Schools, Long Range Planning 33, 621-650.

Bommer, R. und H. Ursprung (1998), Spieglein, Spieglein an der Wand. Eine publikationsanalytische Erfassung der Forschungsleistungen volkswirtschaftlicher Fachbereiche in Deutschland, Österreich und der Schweiz, Zeitschrift für Wirtschafts- und Sozialwissenschaften 118, 1-28.

Brown, B.W. und C.E. Liedholm (2002), Can Web Courses Replace the Classroom in Principles of Microeconomics, American Economic Review 92 (2) (Papers and Proceedings), 444-448.

Burmeister, K.H. (1998), 100 Jahre HSG: Geschichte der Universität St. Gallen, Hochschule für Wirtschafts-, Rechts- und Sozialwissenschaften. Stämpfli, Bern.

Butler, J.S., T.A. Finegan und J.J. Siegfried (1994), Does More Calculus Improve Student Learning in Intermediate Micro and Macro Economic Theory?, American Economic Review 84 (2) (Papers and Proceedings), 206-210.

Butler, J.S., T.A. Finegan und J.J. Siegfried (1998), Does More Calculus Improve Student Learning in Intermediate Micro- and Macroeconomic Theory?, Journal of Applied Econometrics 13, 185-202.

Cohn, E. und S. Cohn (1994), Graphs and Learning in Principles of Economics, American Economic Review 84 (2) (Papers and Proceedings), 197-200.

Colander, D. und A. Klamer (1987), The Making of an Economist, Journal of Economic Perspectives 1 (2), 95-111.

Dubs, R. et al. (eds.) (2004), Einführung in die Managementlehre. Haupt, Bern et al.

Egger, S. und T. Dyllick (2010), Studieren an der HSG, Graduate Survey Report 2010, St. Gallen 2010. (http://www.unisg.ch/ /media/Internet/Content/Dateien/Studium/DarumHSG/ HSG_Studierendenbefragung_2010.ashx?fl = en (08/03711).)

Fournas, P. (2010), Quelle identité pour les Grandes Écoles de commerce françaises? Teil 1: Des écoles de,fils a papa' aux grandes écoles de commerce français: L'émergence d'une nouvelle noblesse de robe (1819 - 2000). Doktorarbeit, École polytechnique, Reims. (http://halshs. archives-ouvertes.fr/docs/00/50/36/41/PDF/Fournas.pdf (12/07/11).)

53. Es gibt Autoren, wie z.B. Frey (2010), die davon ausgehen, dass sich das gesamte Wissenschafts- und insbesondere das Universitätssystem grundlegend verändern wird. 


\section{Gebhard Kirchgässner}

Frank, R.H. (2002), The Economic Naturalist: Teaching Introductory Students How to Speak Economics, American Economic Review 92 (2) (Papers and Proceedings), 459-462.

Frey, B.S. (2010), Withering Academia?, Universität Zürich, CESifo Working Paper Nr. 3209, Oktober.

Frey, B.S. und K. Rost (2008), Do Rankings Reflect Research Quality?, CREMA Working Paper Nr. 2008-22, Basel.

Frey, R.L. (1997), Universitäten im Aufbruch. Helbing und Lichtenhahn, Basel.

Gauthier, A. und X. Wauthy (2007), Teaching versus Research: A Multi-Tasking Approach to Multi-Department Universities, European Economic Review 51, 273-295.

Hansen, L. (1991), The Education and Training of Economics Doctorates, Journal of Economic Literature 29, 1054-1087.

Hansen, L., M.S. Salemi und J.J. Siegfried (2002), Use It or Lose It: Teaching Literacy in the Economics Principles Course, American Economic Review 92 (2) (Papers and Proceedings), 463-472.

Hayashima, A. (1995), Zur Gründung der Handelsakademie St. Gallen, Kwansei Gakuin University Annual Studies 44, 47-78.

Kasper, H. (1991), The Education of Economists: From Undergraduate to Graduate Study, Journal of Economic Literature 29, 1088-1109.

Kirchgässner, B. (1988), Die Handelshochschule Mannheim 1907 - 1933, in: J. Wysocki (Hrsg.), Wirtschaft, Finanzen, Gesellschaft: ausgewählte Aufsätze; Festgabe zu seinem 65. Geburtstag/B. Kirchgässner. Thorbecke, Sigmaringen, 500-508.

Kirchgässner, G. (2005), (Why) Are Economists Different?, European Journal of Political Economy 21, 543-562.

Kirchgässner, G. (2009), Die Krise der Wirtschaft: Auch eine Krise der Wirtschaftswissenschaften?, Perspektiven der Wirtschaftspolitik 10, 436-468.

Kirchgässner, G. (2011), Wissenschaftlicher Fortschritt in den Wirtschaftswissenschaften: Einige Bemerkungen, erscheint in: R. Neck und V. Gadenne (Hrsg.), Philosophie und Wirtschaftswissenschaft. Mohr Siebeck, Tübingen.

Krueger, A.O. (1991), Report of the Commission on Graduate Education in Economics, Journal of Economic Literature 29, 1035-1053.

Mankiw, G.M. (2009) Principles of Economics. 5. Auflage, South-Western Cengage Learning, Mason (Ohio).

Morgan, T. (1995), Theory Versus Empiricism in Academic Economics, Challenge NovemberDecember, 46-51.

Osterloh, M. und B.S. Frey (2009), Research Governance in Academia: Are There Alternatives to Academic Rankings, CESifo Working Paper Nr. 2797, München.

Samuelson, P.A. (1948), Economics. Mc Graw Hill, New York 1948; die 17. Auflage ist zusammen mit W.D. Nordhaus im Jahr 2005 bei McGraw Hill, New York erschienen. Deutsche Übersetzung: Volkwirtschaftslehre. Verlag Moderne Industrie, Landsberg.

Thürer, G. (1974), Hochschule St. Gallen für Wirtschafts- und Sozialwissenschaften, 1899 - 1974. Zollikofer und Co., St. Gallen.

Ulrich, H. und W. Krieg (1972), Das St. Galler Management-Modell. Haupt, Bern et al..

Universität St. Gallen (2011), HSG im Portrait: Zahlen und Fakten. St. Gallen. (http://www. kmu.unisg.ch/org/kmu/web.nsf/SysWebRessources/HSG+Zahlen+und+Fakten/\$FILE/ HSG-Zahlen-und-Fakten-Neuauflage-21Februar2011.pdf (08/03/11).)

Vaubel, R. (2010), Lehren aus der Finanzkrise: Problemadäquate Regulierung und internationale Dimension, mimeo, Universität Mannheim, Mai.

Yeomans, K.A. (1968), Statistics for the Social Scientist. Band 1: Introducing Statistics, Band 2: Applied Statistics. Penguin, Harmondsworth.

Zander, G.H. (2004), Gründungen der Handelshochschulen im deutschen Kaiserreich (1898 1919). Dissertation, Universität zu Köln. 
Abstract: After a short sketch of the history of modern business schools in the German speaking countries, their four major activity fields are considered: (i) academic teaching, (ii) scientific research, (iii) consulting and (iv) executive education. While teaching was traditionally dominant, research has gained more importance in recent decades, not only in Economics but also in Management departments. With respect to consulting, we have to distinguish between consulting for governments by economists and for private companies by professors of management. Executive education is mainly a domain of management (and law) departments; economists only play a minor role in this area. We conclude with discussing some of the ethical questions with which Economics and Management departments are confronted today. 